\section{P4.52 THE COST OF DIAGNOSTIC VERSUS SYNDROMIC MANAGEMENT OF SEXUALLY TRANSMITTED INFECTIONS IN THE HIV EPICENTRE}

${ }^{1}$ Katrina J Stime, ${ }^{1}$ Paul K Drain, ${ }^{2}$ Adrian Mindel, ${ }^{2}$ Sunita Panday, ${ }^{3}$ Hope Ngobese, ${ }^{3}$ Noluthando Ngomane, ${ }^{3}$ Ntuthu Dlamini, ${ }^{4}$ Anne Rompalo, ${ }^{1}$ Ruanne Barnabas, ${ }^{2}$ Nigel Garrett. ${ }^{1}$ University of Washington School of Medicine, Seattle, USA; ${ }^{2}$ Centre for The AIDS Programme of Research in South Africa (CAPRISA), Durban, South African Republic; ${ }^{3}$ Prince Cyril Zulu Clinic, Durban Municipality, Durban, South African Republic; ${ }^{4}$ Johns Hopkins University, Baltimore, USA

\subsection{6/sextrans-2017-053264.549}

Introduction Most low- and middle-income countries (LMICs) rely on syndromic management to guide diagnosis and treatment of sexually transmitted infections (STIs). Syndromic management is less expensive but has poor diagnostic accuracy compared to conventional laboratory testing. The goal of this study was to determine the incremental cost of implementing a rapid, near-patient diagnostic care model at an infectious disease centre in Durban, South Africa.

Methods As part of a cohort study to assess diagnostic pointof-care testing with expedited partner therapy (EPT), we compared cost incurred to standard-of-care syndromic management in the same clinic. Patients were tested with GeneXpert CT/ NG assay, OSOM Rapid Trichomonas Test, and microscopy for candida and bacterial vaginosis, followed by specific treatment and EPT. Patients receiving standard-of-care were administered a symptom screen and broad STI treatment. An activity-based micro-costing approach, including time and motion studies, was used to estimate cost per patient treated. Costs were obtained from budgets, invoices, and staff interviews from 2016.

Results The mean incremental cost per patient when going from syndromic to diagnostic management was USD 36.69 (\$28.15 excluding microscopy). The largest costs for diagnostic management came from Cepheid CT/NG cartridges $(\$ 14.37$, $39 \%)$ and nurse time $(\$ 14.19,39 \%)$. Average per-patient medication costs were higher for syndromic management than diagnostic testing (\$11.00 vs. \$1.74). Several costs of syndromic management were not accounted for, including potential misdiagnosis, overtreatment resulting in adverse reactions or drug resistance, lack of awareness of the specific STI, less effective partner notification with a greater risk of reinfection, and HIV acquisition.

Conclusion Diagnostic management cost more than syndromic management per patient visit. However, health consequences of syndromic management should be further evaluated. Accurate, low-cost STI diagnostics are potentially a cost-effective intervention for STI control.

Support: The CAPRISA 083 study was co-funded by the South African Medical Research Council and the NIH (AI116759). Cepheid loaned two 4-module Genexpert machines to the study team free-of-charge, but did not contribute to the preparation of this abstract

\section{P4.53 CONGENITAL SYPHILIS IN VULNERABLE POPULATIONS: CONSULTATION OFFICE IN THE STREET AS STRATEGY}

Lis Aparecida De Souza Neves; Maria Cristina A. Francelin, Annae Lui Sandrin, Fabiana Rezende Amaral, Maria Cristina Bellizzi Garcia; Monica de Arruda Rocha, Ivana Erse Campos. Ribeirão Preto Municipal Health Department, Ribeirão Preto - SP, Brazil
Introduction In the last decade, syphilis has increased incidence in Brazil, necessitating the integration of health care services, surveillance and actions in the community. The objective of this study was to describe the strategy of using the office on the street to deal with congenital syphilis among pregnant users of crack.

Method A case study, in which the actions implemented and the results achieved will be described. Since 2013, Ribeirão Preto has developed a harm reduction project with vulnerable populations focused on pregnant users of crack living in the street, who are carrying syphilis. In 2016, the project was accredited as Consultation Office in the Street Type II, composed of 5 social action agents and a psychologist, supported by professionals from the STI/AIDS Program. Interventions are carried out daily in scenes of drug use and prostitution. The care of the pregnant woman begins with her identification, sensitisation and conduction to the Health Unit,where she is made reception, collection of the exams, vaccination and medical attention. This pregnant woman and her partner are accompanied by the team and conducted to the care, including for the application of Penicillin.There is integration between the maternity hospitals and the Program. In addition to the weekly planning of the actions and discussion of the cases, Conversation are held together with the Health Unit teams to discuss the service to drug users.

Results Between 2013 and 2016, 30 women were followed up (3 with 2 pregnancies), all crack users, 19 with up to 30 years of age; $30.5 \%$ with more than 5 pregnancies. Nobody was diagnosed with HIV; $76.7 \%$ were with syphilis, all treated after the intervention. Even with follow-up, some of the newborns were born with syphilis; in $43.3 \%$ of the women were placed contraceptive implant.

Conclusion The challenge is constant- pregnant women heavily dependent on the drug, multiplicity of partners, unprepared health workers. There is a need for continuity of the strategy and work with other institutions to form a network of protection for this population.

\section{\begin{tabular}{l|l} 
P4.54 ABSTRACT WITHDRAWN AT THE AUTHOR'S REQUEST \\
\hline
\end{tabular}}


Conclusion Among MSM HIV uninfected, less than half accepted referral and only $13.8 \%$ enrolled in the PrEP program. This highlights the need to strengthen the initial steps of the cascade, perhaps through social marketing and peer networks to enhance awareness and acceptance of PrEP, particularly among high risk communities.

\section{P4.56 EVALUATION OF THREE DNA EXTRACTION METHODS FOR TRICHOMONAS VAGINALIS DIAGNOSIS}

Maria Lucia Gallo Vaulet; S Touzon, Gallo Vaulet MI, M Losada, A Famiglietti, B Perazzi, M Rodriguez Fermepin. Universidad de Buenos Aires, Facultad de Farmacia y Bioquímica, Depto. Bioquímica Clínica, Buenos Aires, Argentina

\subsection{6/sextrans-2017-053264.553}

Introduction Trichomonas vaginalis (TV) is the most prevalent sexually transmitted parasite worldwide. Trichomoniasis is associated with an increased risk of acquiring other sexually transmitted infections and in pregnant women is associated with premature rupture of membranes and preterm delivery. It is important to have high sensitivity diagnostic methods in order to establish appropriate treatments and avoid complications, since approximately $10 \%-50 \%$ of infected women remain asymptomatic. The aim of this study was to evaluate three DNA extraction methods to optimise the detection of TV by PCR.

Methods Vaginal swabs were studied by culture in liquid medium (modified thyoglicolate medium). An aliquot of the original samples was saved for DNA purification by a) using a silica-membrane-based DNA purification commercial kit, b) $10 \mathrm{~min}$ boiling and c) $10 \mathrm{~min}$ boiling followed by sample dilution. All extracts were analysed by PCR for TV (18S rRNA gene). PCR inhibitors were evidenced by human tnf gene amplification. Samples that resulted TV positive by culture and/or PCR were considered as true positive (expanded gold standard).

Results Fortythree vaginal swabs were included in this study. PCR inhibitors were detected in 1 sample prepared by method a), in 2 samples prepared by method b) and c) hence not further analyse. By culture five samples were positive (12.2\%). TV was detected by PCR in a) 12 samples $(29.3 \%)$ b) 7 samples $(17.1 \%)$ and c) 8 samples (19.5\%). All positive culture samples were detected by method a) and c) and only 4 of them by method b). Considering the expanded gold standard, sensitivity for the TV detection by culture was $41.7 \%$, by method b) $58.3 \%$, c) $66.7 \%$ being a) the most sensitive (100\%).

Conclusion Currently the TV molecular diagnosis is not routinely performed and there are no standardised molecular detection methods. Considering the high percentage of asymptomatic patients, the use of high sensitivity techniques such as method a) will allow the improvement of diagnostic protocols and the design of prevention and control strategies. (4) 162 (41.5\%) of them agreed to referred to a PrEP PN, $108(27.7 \%)$ met with PN, and 54 (13.8\%) started PrEP. The majority (70\%) of patients who started PrEP were AA, and there was no difference in uptake between AA MSM and MSM of other race/ethnicity $(p=0.23)$. Among 24 patients enrolled for 6 months or more, 23 (96\%) were retained in PrEP care at 3 months, and $16(67 \%)$ at 6 months. Among these patients, 3 (13\%) were diagnosed with an STD (GC, CT or Syphilis) during follow up and none were infected with HIV. Two out of the 3 patients diagnosed with an STD were among the group that discontinued PrEP. 